\title{
Formation scenarios and mass-radius relation for neutron stars
}

\author{
J. L. Zdunik and P. Haensel
}

\author{
N. Copernicus Astronomical Center PAS, Bartycka 18, 00-716 Warszawa, Poland \\ e-mail: [jlz; haensel]@camk.edu.pl
}

Received 20 October 2010 / Accepted 28 March 2011

\begin{abstract}
Aims. A neutron star crust, which is formed by accretion of matter from a companion in a low-mass X-ray binary (LMXB), has a stiffer equation of state (EOS) than that of catalyzed matter. At a given neutron star mass, $M$, the radius of a star with an accreted crust is therefore larger by $\Delta R(M)$ than for the usually considered star built of catalyzed matter.

Methods. Using a compressible liquid drop model of nuclei, we calculated, within the one-component plasma approximation, the EOSs corresponding to different nuclear compositions of the ashes of X-ray bursts in LMXB. These EOSs are then applied to study the effect of different formation scenarios on the neutron-star mass-radius relation.

Results. Assuming the SLy EOS for neutron star's liquid core, we find that at $M=1.4 M_{\odot}$ the star with accreted crust has a radius more than $100 \mathrm{~m}$ larger than for the crust of catalyzed matter. Using smallness of the crust mass compared to $M$, we derive a formula that relates $\Delta R(M)$ to the difference in the crust EOS. This very precise formula also gives analytic dependence of $\Delta R$ on $M$ and $R$ of the reference star built of catalyzed matter. The formula is valid for any EOS of the liquid core. Rotation of neutron star makes the $\Delta R(M)$ larger. We derive an approximate but very precise formula that gives the difference in equatorial radii, $\Delta R_{\mathrm{eq}}(M)$, as a function of stellar rotation frequency.
\end{abstract}

Key words. dense matter - equation of state - stars: neutron

\section{Introduction}

Establishing the mass versus radius relation for neutron stars by observations is crucial for determining the equation of state (EOS) of dense matter. It is expected that finding even a few points of the $M(R)$ curve could severely limit the range of considered theoretical models of matter in a liquid neutron star core. The general method of determining the EOS at supranuclear densities (i.e. higher densities than the normal nuclear density $\rho_{0}=2.7 \times 10^{14} \mathrm{~g} \mathrm{~cm}^{-3}$ ) from the $M(R)$ curve was developed by Lindblom (1992). A simplified method of determining a supranuclear segment of the EOS from three measured points of the $M(R)$ has recently been described in (Özel et al. 2010). In these studies, the EOS of neutron star crust is considered as well established and fixed. This assumption is valid, within relatively small uncertainties, provided the crust matter is in full thermodynamical equilibrium (catalyzed matter, corresponding at $T=0$ to the ground state of the matter). Such a condition is not fulfilled for the crust formed by accretion of matter onto a neutron star surface, from a companion in a low-mass X-ray binary (accreted crust, Haensel \& Zdunik 1990a,b). The EOS of accreted crust is stiffer than for the ground-state one (Haensel \& Zdunik 1990b). Therefore, at the same stellar mass, $M$, the radius of the neutron star with an accreted crust is larger by $\Delta R(M)$ than that of the star with crust built of catalyzed matter (catalyzed crust). We expect that the millisecond pulsars, spun-up by accretion in LMXBs (Bhattacharya \& van den Heuvel 1991) have accreted crusts, different from the catalyzed crusts of pulsars born in supernova explosions.

We study the effect of the formation scenario on the radiusmass relation for neutron stars. Formation scenarios and corresponding equations of state of the crust are presented in Sect. 2. In Sect. 3 we calculate $\Delta R(M)$ for nonrotating stars, assuming different crust formation scenarios. Numerical results for $\Delta R(M)$, at fixed EOS of the liquid core, are presented in Sect. 3.1. In Sect. 3.2 we derive an approximate, but very precise, general formula that relates the difference in radii to the difference in the EOSs of the crust. The dependence of $\Delta R(M)$ on the EOS of the core enters via a dependence on the mass and radius of the reference star with catalyzed crust. Rotation of neutron star increases $\Delta R(M)$, and in Sect. 4 we study $\Delta R(M)$ (defined as the difference between the equatorial radii) for rotating neutron stars. Section 5 contains a summary of our results, discussion, and conclusion.

Calculations that led to the present note were inspired by questions from the audience during a talk by one of the authors (P.H.) at the CompStar Workshop "Neutron star physics and nuclear physics", held at GANIL, Caen, France, February 14-16, 2010.

\section{Scenarios and equations of state}

\subsection{Catalyzed crust}

We assume that a neutron star was born in a core-collapse supernova. Initially, the outer layers of the star are hot and fluid. Their composition corresponds to nuclear equilibrium, because all reactions are sufficiently rapid at $T \gtrsim 10^{10} \mathrm{~K}$. The crust solidifies in the process of cooling in a newly born neutron star. One assumes that, while cooling and solidifying, the outer layers keep the nuclear equilibrium, and after reaching the strong degeneracy limit, the state of the EOS of the crust is approximated well by the EOS of the cold catalyzed matter, corresponding (in the $T=0$ limit) to the ground of the matter (for a detailed discussion, see, e.g., Haensel et al. 2007). This EOS of the crust will be denoted as EOS[cat]. 


\subsection{Accreted crusts}

We assume that this neutron star has remained $\sim 10^{8}-10^{9} \mathrm{yr}$ in an LMXB. Its crust was formed via accretion of matter onto the neutron-star surface from a companion in the binary. Typical accretion rates in LMXBs are $\sim 10^{-10}-10^{-9} M_{\odot} \mathrm{yr}^{-1}$. Therefore, as a result of the accretion stage, the original, catalyzed crust formed in scenario described in Sect. 2.1 (typical mass of $\sim 10^{-2} M_{\odot}$ ) has been fully replaced by the accreted one.

Accretion and $X$-ray bursts. Many LMXBs are sites of type I $\mathrm{X}$-ray bursts (hereafter: X-ray bursts), which are thermonuclear flashes in the surface layers of an accreting neutron star (Woosley \& Taam 1976; Maraschi \& Cavaliere 1977; Joss 1977). In the simplest model of the X-ray bursts, accreted matter, composed mostly of ${ }^{1} \mathrm{H}$, accumulates on the star surface and undergoes compression from the weight of the continuously accreting matter. The accreted layer is also heated by the plasma hitting the star surface and transforming its kinetic energy into heat. The compressed and heated hydrogen layer burns steadily in its bottom shell with $\rho \sim 10^{5} \mathrm{~g} \mathrm{~cm}^{-3}$ into ${ }^{4} \mathrm{He}$. The helium produced in the burning of hydrogen accumulates in a growing He layer beneath the H-burning shell. After some recurrence time (typically $\sim$ hours), the helium is ignited at the bottom of the He layer, typically at $\rho \sim 10^{6} \mathrm{~g} \mathrm{~cm}^{-3}$. The helium burning starts in a strongly degenerate plasma (temperature $\sim 10^{8} \mathrm{~K}$ and $\rho \sim 10^{6} \mathrm{~g} \mathrm{~cm}^{-3}$ ). Therefore, helium burning is thermally unstable and proceeds initially in an explosive detonation mode, with local temperature exceeding $10^{9} \mathrm{~K}$, and burns the overlaying helium and hydrogen layers into elements with mass number $A \sim 50-100$ (see next paragraph). Finally, the thermonuclear explosion develops into a thermonuclear flash of the surface layer, observable as an X-ray burst. After a few minutes, the H-He envelope has been transformed into a layer of nuclear ashes. The energy released in thermonuclear burning has been radiated in an X-ray burst. Continuing accretion again leads to the accumulation of the $\mathrm{H}-\mathrm{He}$ fuel for the next X-ray burst, and the cycle accretion-burst repeats in a quasi-periodic way every few hours.

Ashes of the X-ray burst. The composition of ashes from thermonuclear burning of an accreted H-He layer deserves a more detailed discussion (see, e.g., Beard \& Wiescher 2003). Early calculations have indicated that thermonuclear explosive burning produces mostly ${ }^{56} \mathrm{Ni}$, which then converts into ${ }^{56} \mathrm{Fe}$ by the electron captures (Taam 1982; Ayasli \& Joss 1982). This picture had to be revised in the light of more recent simulations of nuclear evolution during cooling following the temperature peak of $\sim 2 \times 10^{9} \mathrm{~K}$. These simulations have shown that a few minutes after the initial temperature peak, nuclear ashes contain a mixture of nuclei with $A \sim 50-100$ (Schatz et al. 2001).

Reactions in accreting crust. During accretion, the crust is a site of exothermic reactions in a plasma that is far from the catalyzed state. A layer of ashes from X-ray bursts is compressed under the weight of cumulating overlaying accreted layers. The ashes of density over $10^{5} \mathrm{~g} \mathrm{~cm}^{-3}$ are a plasma composed of nuclei immersed in an electron gas. The temperature in the deeper layer of thermonuclear ashes (a few $10^{8} \mathrm{~K}$ at the depth of a few meters) is too low for the thermonuclear fusion to proceed: the nuclei have $Z \sim 30-50$ and the fusion reactions are blocked by the Coulomb barriers. Therefore, the only nuclear processes are those induced by compression of matter. These processes are electron captures and neutron emissions and absorptions. Compression of ashes results in increasing the density of their layer (and its increasing depth below the stellar surface), and leads to the electron captures on nuclei and the neutronization of the matter. To be specific, let us consider a neutron star with
$M=1.4 M_{\odot}$ (Fig. 39 in Chamel \& Haensel 2008). For densities exceeding $\sim 5 \times 10^{11} \mathrm{~g} \mathrm{~cm}^{-3}$ (neutron drip point in accreted crust, at the depth $\sim 300 \mathrm{~m}$ ) electron captures are followed by the neutron emission from nuclei. Therefore, apart from being immersed in electron gas, nuclei become immersed in a neutron gas. At density over $10^{12} \mathrm{~g} \mathrm{~cm}^{-3}$ (depth greater than $\sim 350 \mathrm{~m}$ ), the value of $Z$ becomes so low and the energy of the quantum zero-point motion of nuclei around the lattice sites so high that the pycnonuclear fusion (see, e.g., Shapiro \& Teukolsky 1983) of neighboring nuclei might be possible. This would lead to a further neutronization of the considered layer of accreted matter. With increasing depth and density, the element of matter under consideration becomes more and more neutronized, and the fraction of free neutrons outside nuclei in the total number of nucleons increases. At the density $10^{14} \mathrm{~g} \mathrm{~cm}^{-3}$ (depth $\sim 1 \mathrm{~km}$ ) the crust matter element dissolves into a homogeneous $n-p-e$ plasma, containing only a few percent of protons.

Nuclear composition of accreted crusts. The composition of a fully accreted crust is calculated, assuming a simple model of one-component plasma, as in Haensel \& Zdunik (2003). All the crust, including its bottom layer, is obtained by compression of an initial shell with $\rho=10^{8} \mathrm{~g} \mathrm{~cm}^{-3}$. Two initial values of the mass number of nuclei in the X-ray bursts ashes are assumed, $A_{\mathrm{i}}=56,106$. Thermal effects are neglected. Extensive numerical simulations of the nuclear evolution of multicomponent ashes, assuming a large reaction network, and taking temperature effects into account, were carried out by (Gupta et al. 2007). However, these calculations were restricted to densities less than $10^{11} \mathrm{~g} \mathrm{~cm}^{-3}$, where the EOS is not significantly different from EOS[cat], albeit the nuclear composition is very different from the one for catalyzed matter.

A model of the EOS of a fully accreted crust will be denoted as $\operatorname{EOS}[$ acc. $\mathcal{A}]$, where $\mathcal{A}$ refers to the assumptions underlying the model: composition of X-ray bursts ashes, types of reactions included in the model, etc.

\subsection{Equations of state}

To disentangle the effect of formation scenario from the EOS of the crust, one has to compare the EOSs calculated not only for the same nuclear Hamiltonian, but also for the same model of nuclei and of nuclear matter in nuclei and neutron matter outside nuclei. In this respect, the analysis of Haensel \& Zdunik (1990b) was not correct, because the EOSs of accreted and catalyzed crusts were based on different nuclear models. In the present paper we consistently use the compressible liquid drop model of nuclei of Mackie \& Baym (1977): this model was used in all previous calculations of accreted crusts. We calculated several EOSs of accreted crust corresponding to different $A_{\mathrm{i}}$ of X-ray bursts ashes and for different models of pycnonuclear reaction rates. These EOSs are plotted in Fig. 1, where we also show EOS[cat] for the same model of the nucleon component (nuclei and neutron gas) of dense matter. For $\rho<10^{11} \mathrm{~g} \mathrm{~cm}^{-3}$ all EOSs are very similar, so we only display EOS plots for $\rho>10^{11} \mathrm{~g} \mathrm{~cm}^{-3}$.

Stiffness of the EOS. Significant differences in this property of the EOS exist in the inner crust, from the neutron drip point on and up to $10^{13} \mathrm{~g} \mathrm{~cm}^{-3}$. In this density range EOS[acc] are significantly stiffer than EOS[cat]. The difference already starts at the neutron drip point, which in the accreted crust is found at a higher density. The softening that follows after the neutron drip is much stronger in EOS[cat] than in EOS[acc]. Then, for $\rho>10^{13} \mathrm{~g} \mathrm{~cm}^{-3}$, EOS[acc] converge (from above) to EOS[cat], because at such high density the nuclei play a lesser rôle in the 


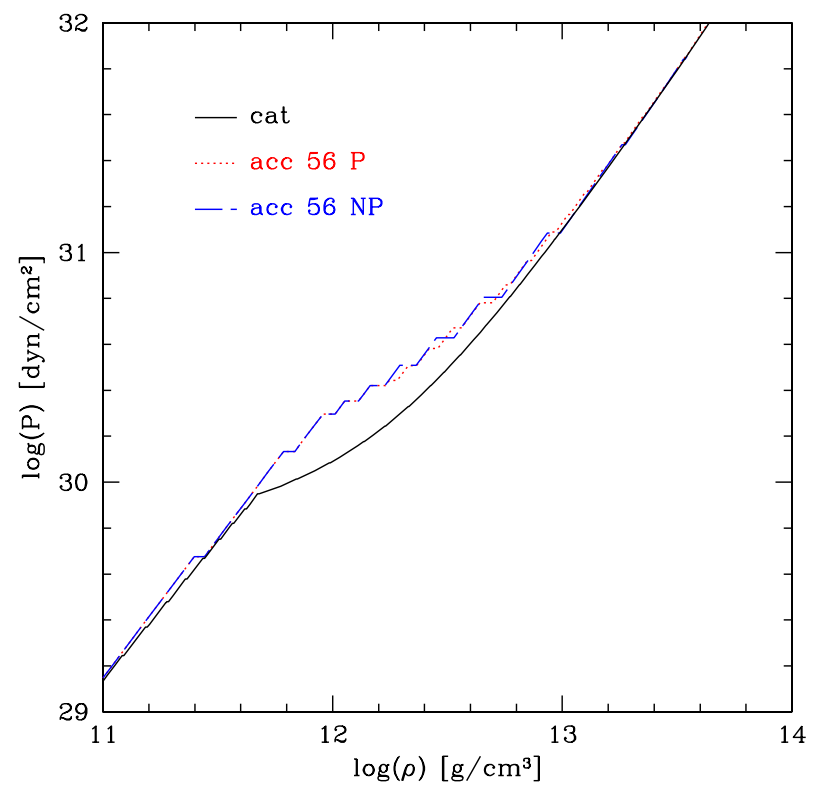

Fig. 1. EOS for the crusts calculated using the Mackie-Baym model of nuclei and neutron matter. Explanation of the labels: cat - cold catalyzed matter; acc.56.P - initial X-ray burst ashes $A_{\mathrm{i}}=56$, pycnonuclear fusion switched on; acc.56.NP - initial X-ray burst ashes $A_{\mathrm{i}}=56$, pycnonuclear fusion switched off till $10^{13} \mathrm{~g} \mathrm{~cm}^{-3}$. For further details see Sect. 2.3. (Color online.)

EOS, and the crust pressure is mostly determined by the neutron gas outside nuclei.

Jumps and smoothness. EOS[acc] shows numerous pronounced density jumps at constant pressure, to be contrasted with the smooth curve of EOS[cat]. Actually, both features are artifacts of the models used. For example, EOS[cat] is completely smooth because we used a compressible liquid drop model without any shell correction for neutrons and protons, and treated neutron and proton numbers within the Wigner-Seitz cells as continuous variables, in which we minimized the enthalpy per nucleon at a given pressure. For EOS[acc] we used one-component plasma approximation, with integer neutron and proton numbers in the Wigner-Seitz cells. Since we neglected thermal effects, we got a density jump at each threshold for electron capture. Had we used a multicomponent plasma model and included thermal effects and a large reaction network, the jumps in the EOS[acc] would be smoothed (see Gupta et al. 2007).

Pycnonuclear fusion. The process of pycnonuclear fusion (see, e.g., Shapiro \& Teukolsky 1983) may proceed after electron captures followed by neutron emission - a reaction chain that results in decreasing $Z$ and $A$. In our simulations based on the one-component plasma model, pycnonuclear fusion proceeds as soon as the time $\tau_{\text {pyc }}$ (inverse of the pycnonuclear fusion rate) is less than the timescale $\tau_{\text {acc }}$ needed for a matter element to pass, owing to accretion, across the (quasistationary) crust shell with specific $(A, Z)$. This time can be estimated as $\tau_{\text {acc }}=M_{\text {shell }}(A, Z) / \dot{M}$. Typical values of the mass of shells in the inner accreted crust are $M_{\text {shell }} \sim 10^{-5} M_{\odot}$. At accretion rates $\dot{M} \sim 10^{-10}-10^{-9} M_{\odot} \mathrm{yr}^{-1}$, formulae used to calculate the fusion rates in (Haensel \& Zdunik 1990a, 2003) lead to pycnonuclear fusions proceeding at $\rho \gtrsim 10^{12} \mathrm{~g} \mathrm{~cm}^{-3}$. However, theoretical evaluation of $\tau_{\text {pyc }}$ is plagued by huge uncertainties (see Yakovlev et al. 2006). It is not certain whether pycnonuclear fusions do indeed occur below $10^{13} \mathrm{~g} \mathrm{~cm}^{-3}$. If they do not, then EOS[acc] is represented quite well by models with pycnonuclear fusion switched off. The two extremes - pycnonuclear fusions

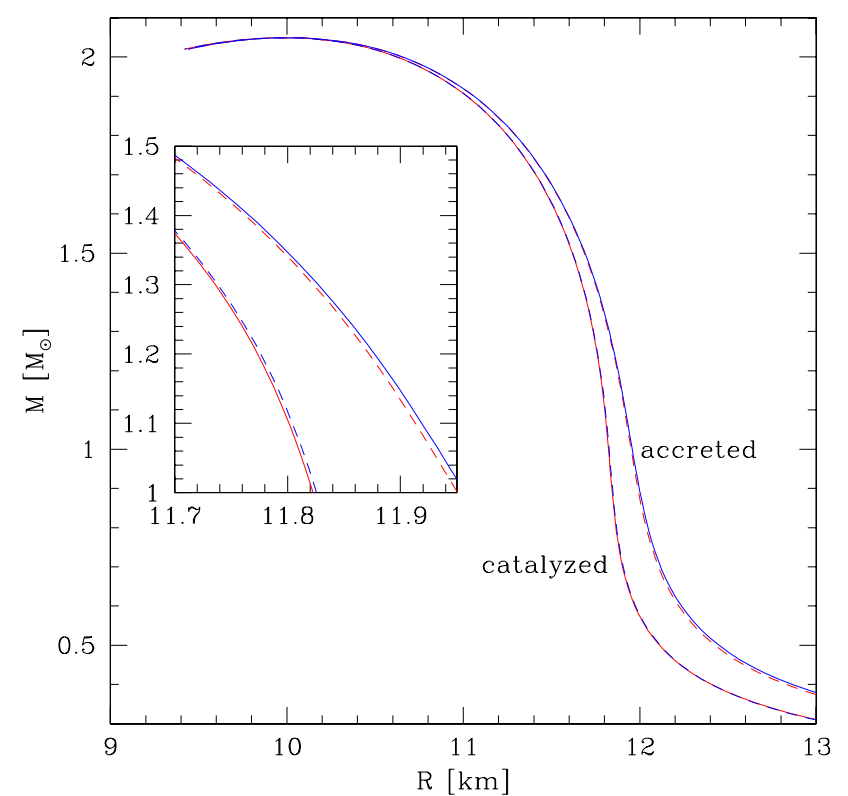

Fig. 2. Solid lines give gravitational mass $M$ versus radius $R$ for nonrotating neutron stars with accreted [acc.56.P] and catalyzed crust. Dashed red lines correspond to the approximation of the crust thickness by Eq. (5). Zoomed region illustrates the very high accuracy of this approximation. For the explanation of the procedure of getting dashed lines, see the last part of Sect. 3.2. (Color online.)

starting at $10^{12} \mathrm{~g} \mathrm{~cm}^{-3}$ and pycnonuclear fusion shifted to densities above $10^{13} \mathrm{~g} \mathrm{~cm}^{-3}$ - correspond to curves EOS[acc. $A_{\mathrm{i}} . \mathrm{P}$ ] and $\operatorname{EOS}\left[\right.$ acc. $\left.A_{\mathrm{i}} . \mathrm{NP}\right]$ in Fig. 1.

Bottom crust layer with $\rho>10^{14} \mathrm{~g} \mathrm{~cm}^{-3}$. Our plots of EOS in Fig. 1 are restricted to $\rho<10^{14} \mathrm{~g} \mathrm{~cm}^{-3}$. For the densities above $10^{14} \mathrm{~g} \mathrm{~cm}^{-3}$ and up to the crust bottom density $\rho_{\mathrm{b}}$, the precision of our models lowers significantly compared to the precision of our EOS at lower densities. Fortunately, the contribution of the bottom layer of the crust $\left(10^{14} \mathrm{~g} \mathrm{~cm}^{-3}<\rho<\rho_{\mathrm{b}}\right)$ to the difference $R_{\text {acc }}-R_{\text {cat }}$ is negligible, and therefore the uncertainties in the crust EOS at the highest densities do not affect our main results. This favorable situation will be justified in Sect. 3.2.

\section{Effect of the change of the crust EOS on the star radius}

\subsection{Accreted vs. catalyzed crust: numerical results for nonrotating models}

We matched EOSs of the crust described in the preceding section to several EOSs of the liquid core. We checked that the difference $\Delta R(M)=R_{\text {acc }}(M)-R_{\text {cat }}(M)$ does not depend on the details of matching in the crust-core interface. On the other hand, $\Delta R(M)$ can be shown to depend on the EOS of the core via the factor $\left(R_{\text {cat }} / r_{\mathrm{g}}-1\right) R_{\text {cat }}$, where $r_{\mathrm{g}}=2 G M / c^{2}=2.96 \mathrm{M} / M_{\odot} \mathrm{km}$ is the gravitational (Schwarzschild) radius. These two properties are derived using the equations of hydrostatic equilibrium in Sect. 3.2.

In Fig. 2 we show the $M(R)$ relation for neutron stars with catalyzed and accreted crust (EOS[acc.56.P]). For the liquid core we use the SLy EOS of (Douchin \& Haensel 2001). For $M=$ $1.4 M_{\odot}$, we get $\Delta R \approx 100 \mathrm{~m}$. The value of $\Delta R$ grows to $200 \mathrm{~m}$ if $M$ decreases to $1 M_{\odot}$. It decreases to $40 \mathrm{~m}$ for $1.8 M_{\odot}$. 


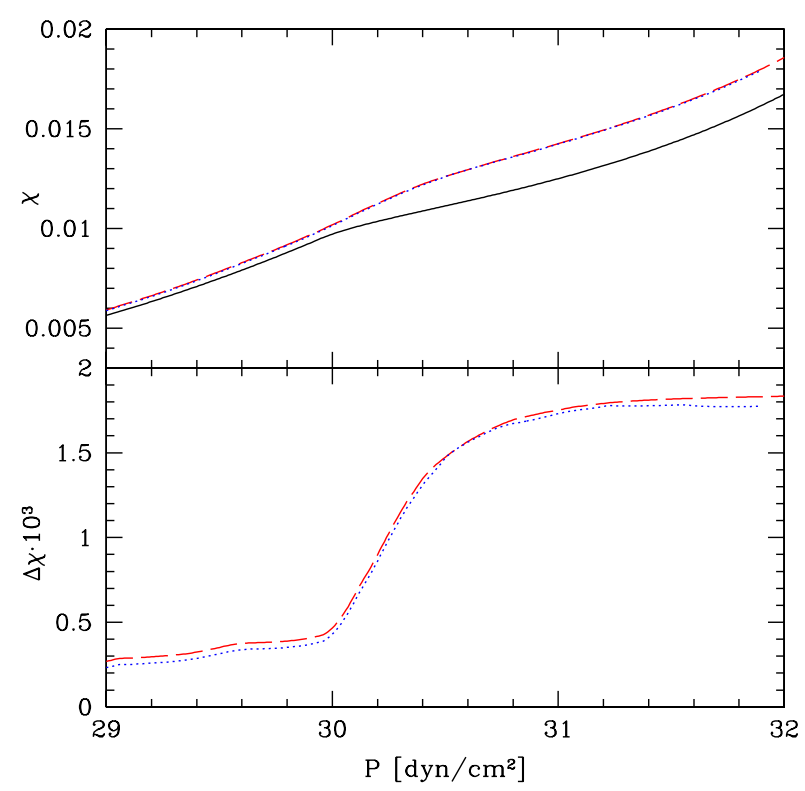

Fig. 3. Upper panel: functions $\chi(P)$ for EOS[acc.56.P] (dashed, red), EOS[acc.106.NP] (dotted, blue), and EOS[cat] (solid, black). Lower panel: function $\Delta \chi(P)($ Eq. (4)) for EOS[acc.56.P] (dashed, red) and EOS[acc.106.NP] (dotted, blue). (Color online.)

\subsection{An analytic approximation for $\Delta R$}

We consider hydrostatic equilibrium of a nonrotating neutron star. The (circumferential) radius of the star is $R$ and its (gravitational) mass $-M$. The pressure at the bottom of the crust is $P_{\mathrm{b}}$, and the mass of the crust is $M_{\mathrm{cr}}$. We assume that $M_{\mathrm{cr}} / M \ll 1$, but we account for the radial extension of the crust and the radial dependence of the pressure and of the density within the crust, following the method formulated in (Zdunik et al. 2008). We use the fact that $P / c^{2} \ll \rho$ and $4 \pi r^{3} P / M c^{2} \ll 1$ within the crust. We define a dimensionless function of pressure within the crust $\left(0 \leq P \leq P_{\mathrm{b}}\right)$

$\chi(P)=\int_{0}^{P} \frac{\mathrm{d} P^{\prime}}{\rho\left(P^{\prime}\right) c^{2}}$.

The function $\chi(P)$ is determined solely by the EOS of the crust.

Using the Tolman-Oppenheimer-Volkoff equation of hydrostatic equilibrium (Shapiro \& Teukolsky 1983), and neglecting within the crust $P / c^{2}$ compared to $\rho$ and to $M /\left(4 \pi r^{3}\right)$, we can go over in Eq. (1) to the radial coordinate $r$, getting

$\chi[P(r)]=\frac{1}{2} \ln \left[\frac{1-r_{\mathrm{g}} / R}{1-r_{\mathrm{g}} / r}\right]$,

where $r_{\mathrm{g}} \equiv 2 G M / \mathrm{c}^{2}$.

The dimensionless function $\chi \ll 1$ can be treated as a small parameter in systematic expansions of the crust thickness. This function increases monotonously with $P$, from zero to $\sim 10^{-2}$ (upper panel of Fig. 3). Therefore, an expression for the crust layer thickness, from the surface to the pressure at the layer bottom $P, \mathrm{t}(P) \equiv R-r(P)$, obtained from Eq. (2) in the linear approximation in $\chi$, is expected to be very precise,

$\mathrm{t}(P)=2 \chi(P)\left(\frac{R}{r_{\mathrm{g}}}-1\right) R$.

Our aim is to evaluate the change in the radius of neutron star of mass $M$ and radius $R$, when EOS[cat] is replaced by EOS[acc]. These EOSs differ for pressures below $P_{1}=10^{32} \mathrm{erg} \mathrm{cm}^{-3}$
(Sect. 2.3 ). To calculate $R_{\text {acc }}-R_{\text {cat }}$ it is therefore sufficient to know the difference in values of $\chi$ at $P_{1}$. We introduce a function $\Delta \chi(P)$, measuring the difference between two EOSs of the crust for pressures from zero to $P$,

$\Delta \chi(P) \equiv \chi_{\mathrm{acc}}(P)-\chi_{\mathrm{cat}}(P)$.

In Fig. 3 we show functions $\chi(P)$ and $\Delta \chi(P)$ for several EOSs of accreted crust. In spite of the jumps in density at constant pressure, characteristic of the EOSs of accreted crusts (Fig. 1), both $\chi$ and $\Delta \chi$ are smooth functions of $P$. This is due to the integration over $P^{\prime}<P$ in the definition of $\chi(P)$. As seen in Fig. 3, dependence of $\chi$ and $\Delta \chi$ on the particular scenario underlying EOS[acc] is very weak. Additionally, $\Delta \chi$ is nearly constant for $P>2 \times 10^{31} \mathrm{erg} \mathrm{cm}^{-3}$, because EOS[cat] and EOS[acc] converge at high pressures (see Fig. 1). Therefore, $\Delta \chi\left(P_{1}\right)$ is a very good approximation for $\Delta \chi\left(P_{\mathrm{b}}\right)$.

Our final formula, obtained using Eqs. (3) and (4), combined with approximations explained above, is

$R_{\mathrm{acc}}-R_{\mathrm{cat}} \simeq 2 \Delta[\chi] \cdot\left(R / r_{\mathrm{g}}-1\right) R$,

where $\Delta[\chi]=\Delta \chi\left(P_{1}\right)$ and $R=R_{\text {cat }}$.

The precision of the approximation (5) is illustrated in Fig. 2. The dashed line for the accreted crust was obtained from exact $R_{\text {cat }}(M)$ using Eq. (5) with $R=R_{\text {cat }}$. On the other hand, the dashed line for catalyzed crust was obtained from exact $R_{\text {acc }}(M)$ using Eq. (5) with $R=R_{\text {acc }}$.

Up to this point, we have neglected the contribution of elastic strain to the stress tensor within the crust, and used the perfect liquid approximation in the equations of hydrostatic equilibrium. Recent numerical simulations indicate that, on the timescales of years and longer (which are of astrophysical interest), the breaking stress of the ion crystal of the crust is $\sim 10^{-3}$ of the crust pressure (Chugunov \& Horowitz 2010). This is much less than the difference between EOS[acc] and EOS[cat]. Therefore, as long as we restrict ourselves to a timescale longer than a few years, the contribution of elastic strain to $\Delta R(M)$ can be neglected.

\section{Combined effects of accretion and rotation on $\Delta R(M)$}

Millisecond pulsars are thought to be recycled old ("dead") pulsars, spun-up by accretion of matter from their companion in LMXBs (Alpar et al. 1982; Bhattacharya \& van den Heuvel 1991). This scenario is corroborated by the discovery of rapid $\mathrm{X}$-ray pulsations with frequencies up to $619 \mathrm{~Hz}$ in more than a dozen LMXBs. The most rapid millisecond pulsar (isolated one) rotates at $716 \mathrm{~Hz}$. To reach such high rotation frequencies, neutron star had to accrete some five times the mass of the crust of a $1.4 M_{\odot}$ neutron star. Therefore, one has to conclude that millisecond pulsars have fully accreted crusts.

In the case of rotating stars, we define $R_{\text {acc }}-R_{\text {cat }}$ as the difference in circumferential equatorial radii. Rotation increases the difference $R_{\text {acc }}-R_{\text {cat }}$ compared to the static case. A rough Newtonian argument relies on the proportionality of the centrifugal force to the distance from the rotation axis. Therefore, centrifugal force that acts against gravity is strongest at the equator. In order to get the precise value of the effect of rotation on $R_{\text {acc }}-$ $R_{\text {cat }}$, we performed 2-D simulations of stationary configurations of rigidly rotating neutron stars with catalyzed and accreted crusts. The calculations were performed using the rotstar code from the LORENE library (http: //www . lorene. obspm. fr). 


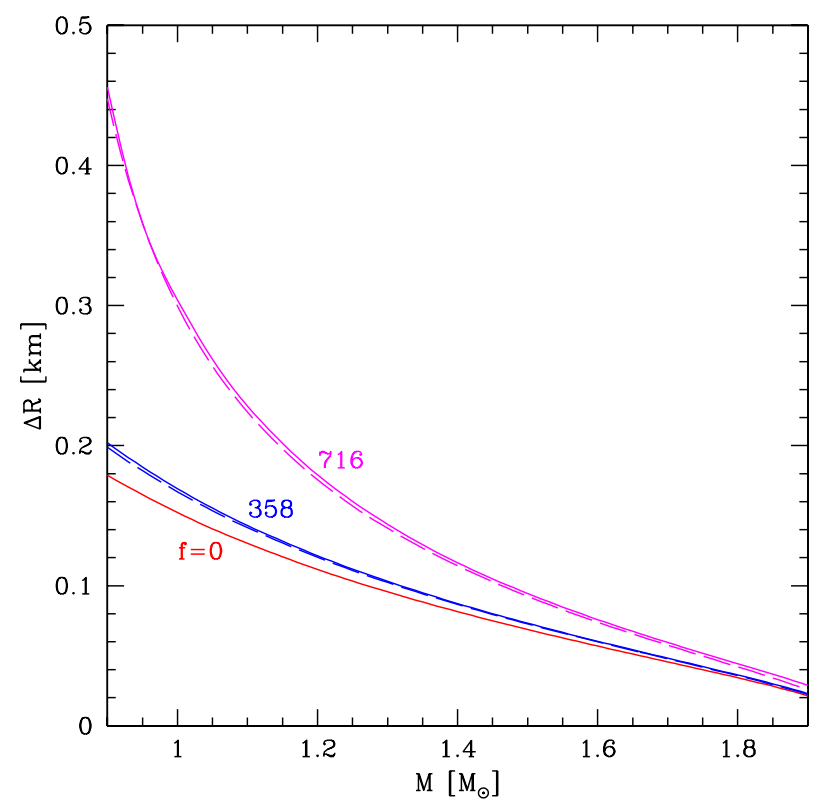

Fig. 4. Dependence of $\Delta R(M)=R_{\text {acc }}(M)-R_{\text {cat }}(M)$ on the frequency of rotation of neutron star, $f$. Equations of state as in Fig. 2. Upper curve $f=716 \mathrm{~Hz}$. Middle curve $-f=358 \mathrm{~Hz}$. Bottom curve - nonrotating.

Our results, obtained for $f=716 \mathrm{~Hz}$ (maximum frequency measured for a pulsar) and for a half of this frequency, are shown in Fig. 4.

Consider hydrostatic equilibrium of the crust of rotating star in the equatorial plane. In the Newtonian approximation, the ratio of centrifugal force to the gravitational pull at the equator is, neglecting rotational deformation, $\Omega^{2} R^{3} / G M$, where $\Omega$ is angular frequency of rigid rotation. This expression is exact in the quadratic approximation in $\Omega$, because an increase of $R$ due to rotation introduces higher powers of $\Omega$. We propose modeling the centrifugal-force effect within crust by modifying the Tolman-Oppenheimer-Volkoff equation in the equatorial plane in following way:

$$
\frac{\mathrm{d} P}{\mathrm{~d} r}=-\frac{G M \rho}{r^{2}}\left(1-\frac{2 G M}{r c^{2}}\right)^{-1}\left(1-\alpha \Omega^{2} R^{3} / G M\right)
$$

where $M$ is the total mass of the star and $\alpha$ a numerical coefficient to be determined by fitting the exact results of 2-D calculations. The validity of Eq. (6) relies on the smallness of the rotational flattening of the liquid core compared to that of crust. We included terms quadratic in $\Omega$ and used a standard approximation valid for a low-mass crust, applied in the preceding section. Equation (6) could be solved explicitly by assuming constant $M$ and taking the changes in $r$ throughout the crust into account, as in the case of nonrotating star in (Zdunik 2002). However, to be consistent with the approach leading to formula (3), we prefer to use a solution method based on the smallness of $\chi$. The rotation effect enters via a constant factor $\left(1-\alpha \Omega^{2} R^{3} / G M\right)$. This results in a simple relation between $\Omega=0$ and $\Omega>0$ difference in radii:

$$
\begin{aligned}
\Delta R_{\mathrm{eq}}(\Omega) & =R_{\mathrm{eq}, \mathrm{acc}}(\Omega)-R_{\mathrm{eq}, \mathrm{cat}}(\Omega) \\
& =\frac{2 \Delta[\chi] \cdot\left(R / r_{\mathrm{g}}-1\right) R}{1-\alpha \Omega^{2} R^{3} / G M}=\frac{\Delta R(\Omega=0)}{1-\alpha \Omega^{2} R^{3} / G M} .
\end{aligned}
$$

The dimensionless parameter $\alpha$ has been determined numerically by fitting formula (7) to the exact $2-\mathrm{D}$ results obtained in General Relativity using LORENE numerical library. We find that $\alpha \approx 4 / 3$. As one sees in Fig. 4, our approximate formula for $\Delta R_{\text {eq }}(\Omega)$ works extremely well. The actual $\Omega$-dependence of $\Delta R_{\text {eq }}$ is stronger than quadratic because $\Omega^{2}$ appears in the denominator in Eq. (7).

\section{Summary and conclusion}

We have studied the effect of the formation scenario on the massradius relation for neutron stars. For a given $M$, a star with an accreted crust has a larger radius by $\Delta R(M)$, than a star built of catalyzed matter formed in stellar core collapse. We derived an approximate but very precise formula for $\Delta R(M)$, valid for slowly rotating neutron stars. $\Delta R(M)$ factorizes into a prefactor depending solely on the EOS of neutron star crusts formed in different scenarios and a simple function of $M$ for a given EOS of the core. We studied the dependence of the difference between the equatorial radii on the angular rotation frequency, $\Delta R_{\text {eq }}(\Omega)$. We derived an approximate formula for $\Delta R_{\mathrm{eq}}(\Omega)$, which reproduces $\Delta R_{\text {eq }}$ with high precision even for neutron stars rotating at $716 \mathrm{~Hz}$, the highest rotation frequency measured for a radio pulsar.

We found that an accreted crust makes the radius of a $2 M_{\odot}-1 M_{\odot}$ star some 50-200 m larger than in the standard catalyzed matter case. The highest hopes of a simultaneous measurement of a neutron star $M$ and $R$ are, in this decade, associated with high-resolution X-ray spectroscopy (Arzoumanian et al. 2009; Paerels et al. 2009). Unfortunately, expected uncertainty in determining $R(M)$ is $\pm 5 \%$. It significantly exceeds the effects of formation scenarios, as calculated here.

Acknowledgements. This work was partially supported by the Polish MNiSW grant No. N N203 512838. This work was also supported in part by CompStar, a Research Networking Program of the European Science Foundation and the LEA Astro-PF.

\section{References}

Alpar, M. A., Cheng, A. F., Ruderman, M. A., \& Shaham, J. 1982, Nature, 728 Arzoumanian, Z., et al. 2009, in Astro2010: The Astronomy and Astrophysics Decadal Survey, White paper no. 6 [arXiv:0902 . 3264]

Ayasli, S., \& Joss, P. C. 1982, ApJ, 256, 267

Beard, M., \& Wiescher, M. 2003, Rev. Mex. Fisica, 49, 139

Bhattacharya, D., \& van den Heuvel, E. P. J. 1991, Phys. Rep., 203, 1

Chamel, N., \& Haensel, P. 2008, Liv. Rev. Rel., 11, 10

Chugunov, A. I., \& Horowitz, C. J. 2010, MNRAS, 407, L54

Douchin, F., \& Haensel, P. 2001, A\&A, 380, 151

Gupta, S., Brown, E. F., Schatz, H., Möller, P., \& Kratz, K.-L. 2007, ApJ, 662, 1188

Güver, T., Özel, F., Cabrera-Lavers, A., \& Wroblewski, P. 2010, ApJ, 712, 964

Haensel, P., \& Zdunik, J. L. 1990a, A\&A, 227, 431

Haensel, P., \& Zdunik, J. L. 1990b, A\&A, 229, 117

Haensel, P., \& Zdunik, J. L. 2003, A\&A, 404, L33

Haensel, P., \& Zdunik, J. L. 2008, A\&A, 480, 459

Haensel, P., Potekhin, A. Y., \& Yakovlev, D. G. 2007, Neutron Stars 1, Equation of State and Structure (New York: Springer)

Joss, P. C. 1977, Nature 270, 310

Lindblom, L. 1992, ApJ, 398, 569

Mackie, F. D., \& Baym, G. 1977, Nucl. Phys. A, 285, 332

Maraschi, L., \& Cavaliere, A. 1977, in Highlights of Astronomy, ed. E. A. Mueller (Dordrecht: Reidel), Part I, 4, 127

Özel, F. 2006, Nature, 441, 1115

Özel, F., Güver, T., \& Psaltis, D. 2009, ApJ, 693, 1775

Özel, F., Baym, G., \& Güver, T. 2010, Phys. Rev. D, 82, 101301

Paerels, F., M. Mendez., M., Agueros, et al. 2009, in Astro2010: The Astronomy and Astrophysics Decadal Survey, White paper no. 230 [arXiv:0904.0435]

Schatz, H., Aprahamian, A., Barnard, V., et al. 2001, Phys. Rev. Lett., 86, 3471

Shapiro, S. L., \& Teukolsky, S. A. 1983, Black Holes, White Dwarfs, and Neutron Stars: The Physics of Compact Objects (New York: Wiley)

Taam, R. E. 1982, ApJ, 258, 761

Woosley, S. E., \& Taam, R. E. 1976, Nature, 263, 101

Yakovlev, D. G., Gasques, L., \& Wiescher M. 2006, MNRAS, 371, 1322

Zdunik, J. L. 2002, A\&A, 394, 641

Zdunik, J. L., Bejger, M., \& Haensel, P. 2008, A\&A, 491, 489 